\title{
Original
}

\section{Factores pronósticos del carcinoma de células renales}

\author{
Rafael A. Medina López, José M. Conde Sánchez, Carmen B. Congregado Ruiz, \\ Raquel González Resina, Salvador Mármol Navarro, Francisco J. Torrubia Romero
}

Servicio de Urología. Hospital Universitario Virgen del Rocío. Sevilla, España.

\begin{abstract}
Resumen
El diagnóstico del cáncer renal ha aumentado en las últimas décadas. A esto hay que sumar la diversidad morfológica de las neoplasias renales, llegando a plantearse la existencia de una gran variedad de entidades bajo el concepto de carcinoma renal (CR). El estudio EROCARE-4, publicado recientemente, muestra una supervivencia global a los 5 años del $59,2 \%$. En este contexto, y como ocurre en toda patología oncológica, la determinación adecuada de factores pronósticos ayudaría a plantear la estrategia terapéutica más apropiada en cada caso, dirigir futuros tratamientos y desarrollar esquemas específicos de seguimiento para nuestros pacientes. En la actualidad los diferentes factores pronósticos de CR podemos clasificarlos en cuatro grupos: anatómicos, clínicos, histológicos y moleculares. En este trabajo analizamos estos factores pronósticos y revisamos los nomogramas más extendidos en la actualidad.
\end{abstract}

Palabras clave: Carcinoma de células renales. Factores pronósticos. Supervivencia.

\section{Prognostic factors in renal cell carcinoma}

\section{Abstract}

Renal cancer has been increasingly diagnosed in recent decades. In addition, morphological diversity of renal neoplasms has led to consider that the concept of renal cell (RC) carcinoma encompasses a wide variety of conditions. The recently publshed EROCARE-4 study showed a 5-year overall survival rate of 59.2\%. In this and any other neoplastic disease, determination of adequate prognostic factors would help decide the most appropriate therapeutic strategy in each case, guide future treatments, and develop specific follow-up schemes for our patients. The diferent prognostic factors for RC may currently be classified into four groups: anatomical, clinical, histological and molecular. This paper analyzes these prognostic factors and reviews the nomograms most commonly used.

Keywords: Renal cell carcinoma. Prognostic factors. Survival rates.

$\mathrm{E}$ diagnóstico del cáncer renal ha aumentado en las últimas décadas debido a diferentes motivos, entre ellos al avance en las técnicas diagnósticas. En este sentido, la estimación realizada por la Agencia Internacional de Investigación del Cáncer (IARC) para la prevalencia del cáncer de riñón en nuestro país fue de 2.977 casos en 1 año, 8.273 casos en 3 años y 12.079 casos en 5 años. La incidencia/100.000 habitantes se situaría en 13,97 para los hombres y 4,83 para las mujeres en el periodo 1997-2000, según el análisis publicado por el Ministerio de Sanidad en el año $2005^{1}$. La tasa cruda de mortalidad/100.000 habitantes se sitúa en 5,7 para los hombres y 2,9 para las mujeres.

El estudio EROCARE-4, desarrollado en Europa y publicado recientemente, muestra una supervivencia global a los 5 años del 59,2\%².
Otro aspecto a destacar es el hecho de que el 30\% de estos tumores son metastáticos a su diagnóstico ${ }^{3}$, y que los pacientes sometidos a tratamiento quirúrgico en estadios localizados el 30\% desarrollarán metástasis a lo largo de su evolución. A esto hay que sumar la diversidad morfológica de las neoplasias renales, llegando a plantearse la existencia de una gran variedad de entidades bajo el concepto de carcinoma renal (CR), con un comportamiento biológico diferente cada una de ellas, y con base citogenética individual ${ }^{4}$.

En este sentido, podemos decir que en nuestros días se priorizan tres líneas de actuación en el $\mathrm{CR}^{5}$ :

- Detección en estadios precoces.

- Desarrollo de terapias sistémicas más efectivas para la enfermedad metastásica.

- Identificación de nuevos marcadores que aporten información sobre el pronóstico y respuesta a los diferentes tratamientos. 
Con todo, la determinación adecuada de factores pronósticos ayudaría a plantear la estrategia terapéutica más apropiada en cada caso, dirigir futuros tratamientos y desarrollar esquemas específicos de seguimiento para nuestros pacientes.

Podemos clasificar los diferentes factores pronósticos estudiados hasta el momento en 4 gru$\operatorname{pos}^{6,7}$ (Tabla 1):

1. Anatómicos.

2. Clínicos.

3. Histológicos.

4. Moleculares.

Con ellos, diferentes grupos de trabajos han desarrollado nomogramas, a modo de algoritmos pronósticos, con el objetivo de facilitar la toma de decisiones en el paciente con CR.

En este trabajo analizamos estos factores pronósticos y revisamos los nomogramas más extendidos en la actualidad.

\section{FACTORES ANATÓMICOS}

En este grupo de factores encontramos a aquellos directamente relacionados con el tamaño tumoral y su extensión, tanto local, ganglionar, vascular, y/o metastásica.

Tabla 1. Factores pronósticos carcinoma renal

Anatómicos
Tamaño tumoral
Extensión tumoral
$\quad$ Afectación cápsula renal
$\quad$ Afectación sistema colector
$\quad$ Afectación suprarrenal
$\quad$ Afectación linfática
$\quad$ Afectación vascular
Metástasis a distancia
Clinicos
Sintomatología
Performance status
Trombocitosis
Caquexia
Neutrofilia
Histológicos
Grado tumoral
Tipo histológico
Rasgo sarcomatoide
Necrosis
Moleculares
VE IX

CA IX: carbonic anhydrase IX; VEGF: vascular endotelial growth factor.
Estos factores quedan globalmente recogidos en la clasificación TNM $2002^{8}$ y por tanto en el sistema de estadiaje comúnmente utilizado en la practica diaria.

\section{Estadio tumoral}

Como es evidente, los pacientes con tumores organoconfinados presentan un mejor pronóstico en relación a los que sufren enfermedad diseminada. Evaluando la implicación pronóstica del estadiaje establecido por la clasificación TNM, un análisis multivariante publicado en 2000 muestra que la supervivencia se encuentra significativamente relacionada con él. De esta forma, la supervivencia cáncer específica a los 5 años para los pacientes en estadio I se sitúa en el 94\%, 74\% para el II, 67\% para el III y $32 \%$ para el estadio IV $^{9}$.

En relación a este sistema y sus implicaciones pronósticas han surgido diferentes críticas, sugiriendo su reevaluación, dada la discrepancia en los límites marcados para el tamaño tumoral en comparación con estudios más recientes, y la importancia fundamental de la existencia de trombo tumoral, afectación perirrenal y de la grasa del seno, así como de la afectación adrenal y ganglionar, al margen de no incluir otros factores, clínicos e histológicos, vitales para el pronóstico, como veremos a lo largo de este trabajo ${ }^{5,10}$.

\section{Tamaño tumoral}

Siguiendo lo pautado en la clasificación TNM 2002, el límite de $4 \mathrm{~cm}$ clasificará al tumor como T1a o T1b, con las correspondientes implicaciones en las decisiones quirúrgicas y de seguimiento posterior, dado que la seguridad y eficacia de la cirugía ahorradora de nefronas, según estudios multicéntricos, parece demostrada para tumores menores de 4 $\mathrm{cm}^{11}$. En este sentido, existe un estudio de validación con 2.746 pacientes que establece diferencias de supervivencia cáncer-específica a los 5 años en relación al tamaño tumoral, situándola en el 97\% para los Tla $(7 \mathrm{~cm})^{12,13}$.

Por otro lado, emerge una corriente que aboga por elevar el límite del tamaño tumoral de 4 hasta 7 cm para la decisión de cirugía conservadora en base a la ausencia de diferencias pronósticas significativas. Un estudio publicado por Leibovich et al. que incluía 932 pacientes con CR entre 4 y 7 cm sometidos a tratamiento quirúrgico, no encontró diferencias estadísticamente significativas entre los pacientes 
sometidos a cirugía conservadora o radical, por lo que concluye que la cirugía conservadora en paciente con este tamaño tumoral no ensombrece el pronóstico ${ }^{14}$.

Un estudio multicéntrico realizado en Europa por Ficarra et al, defiende situar el corte en $5,5 \mathrm{~cm}$ en base a implicaciones pronósticas, dadas las diferencias encontradas, estadísticamente significativas, en la supervivencia específica a los 5 y 10 años, que resultaron ser del 93,2\% y 87,9\% respectivamente para los $=5,5 \mathrm{~cm}$, y del 79,95 y 73,5\% para los $>5,5 \mathrm{cms}(\mathrm{p}<0,0001)^{15,16}$.

En nuestro país, encontramos igualmente disparidad en los resultados publicados. Existen grupos que en el análisis de su serie no encuentran diferencias en la supervivencia entre los T1a/b y los T2, situándolas entre el 95 y 100\% a los 5 años ${ }^{17,18}$. Por el contrario, otros sí aprecian diferencias significativas, con una supervivencia a los 5 años entre el 95 y 100\% para los T1a, 87\% para los T1b y del 66- 79,9\% para los T2 ${ }^{19,20}$.

En líneas generales, en estudios multivariantes, el tamaño tumoral suele mostrarse como una variable pronóstica independiente ${ }^{21}$.

\section{Extensión tumoral}

Es evidente que el grado de extensión del CR compromete su pronóstico. Múltiples estudios muestran datos sobre las diferencias pronósticas en relación a la afectación de la cápsula renal, del sistema colector, de la grasa perirrenal/glándula suprarrenal (T3a), vascular (T3b-c) o ganglionar (N1-2).

\section{Afectación de la cápsula/sistema colector}

En un estudio publicado el pasado año ${ }^{22}$, se analizó la influencia pronóstica de estos dos factores en los tumores en estadio I y II en una serie de 519 casos, llegando a la conclusión, mediante un análisis multivariante, que la afectación de la cápsula renal (invasión pero sin penetración) y del sistema colector son factores pronósticos independientes, hasta tal punto que cuando existe alguna de ellas la supervivencia de los T1NOMO y los T2NOMO llega a no alcanzar diferencias significativas en relación a los T3aNOMO.

En relación a la invasión capsular, la proporción de pacientes libres de enfermedad a los 5 años varía del 75,6\% de aquellos con invasión al 86,9\% en los que no la presentan. En cuanto a la afectación del sistema colector, esta proporción varía del 56.9\% en los que la sufren al $86.3 \%$ en los que no está presente.

\section{Afectación grasa prerrirenal/glándula suprarrenal}

Analizados de forma global, la supervivencia de los CR T3a se sitúa según diferentes grupos entre el $50-75 \%$ a los 5 años ${ }^{13,17}$.

En un estudio realizado por Han et al. en 1.087 pacientes intervenidos, donde analizan por separado la supervivencia de los CR con afectación o no de la glándula suprarrenal, muestran que dicha afectación ensombrece significativamente el pronóstico, encontrando una supervivencia a los 5 años del 36\% para los casos con afectación de la grasa, reduciéndose al $0 \%$ en los casos con afectación adrenal ${ }^{23}$.

\section{Afectación vascular}

La invasión vascular puede ser micro o macroscópica.

La existencia de invasión microscópica se muestra como un factor pronóstico independiente desfavorable $21,24-27$. Posee un pronunciado efecto negativo sobre el periodo libre de recurrencia, incluso en los pacientes con estadios bajos, pudiendo, en estos casos, descender la supervivencia a los cinco años desde el 90\% de aquellos que no la presentan hasta el $45 \%$ de los que la poseen.

Según el estudio de Van Poppel et $\mathrm{al}^{24}$ el 28,3\% de los pacientes con CR incluidos en su serie, presentaban invasión microscópica vascular, de ellos el $39,2 \%$ presentaron progresión de la enfermedad, con un intervalo libre de 72 meses. De los casos sin invasión vascular, sólo el 6,2\% experimentaron progresión, con un intervalo libre de 160 meses.

En relación al desarrollo de metástasis, un 50\% de los afectados por invasión microvascular las desarrolla frente al 5\% de los casos con ausencia de la misma ${ }^{27}$.

La afectación vascular macroscópica viene dada por la tendencia del CR a crecer intraluminalmente en el sistema venoso, fundamentalmente hacia la vena renal y cava inferior. Puede afectar en torno al $10 \%$ de los casos al diagnóstico ${ }^{28,29}$, y su existencia clásicamente se ha asociado a mayor recurrencia y disminución de la supervivencia tras la cirugía, aunque existen publicaciones en pro y en contra de esta afirmación.

De esta forma, Kim et al. muestran que a los 3 años el 78\% de los casos sin afectación vascular sometidos a nefrectomía se encontrarían libres de enfermedad, frente al 60\% de los que presentaban trombo en vena renal, el 46\% si afectaba la cava infradiafragmática y el 34\% si presentaban trombo en cava supradiafragmática y se realizó cirugía sobre el mismo ${ }^{28}$. 
Por otro lado, un reciente estudio publicado por Lambert et al. muestra ausencia de significación estadística en la supervivencia cáncer-específica a los cinco años en relación al nivel del trombo. La mayor diferencia la marcaría la existencia o no de metástasis asociadas, con una buena supervivencia a los 5 años tras el tratamiento quirúrgico $(60,3 \%$ frente al $10 \%$ sin o con metástasis respectivamente) ${ }^{30}$. En esta misma línea, Boorjian et al. comunican una supervivencia a los 5 años del 44,6\% en los pacientes con trombo en vena renal/cava infradiafragmática (T3b) y del 47,4\% en los que presentaban trombo supradiafragmático ${ }^{31}$.

Muy probablemente estos resultados tengan una estrecha relación con la experiencia del equipo en esta compleja cirugía, y siempre en ausencia de invasión de la pared vascular por el trombo, dado que este hecho empobrecería el pronóstico descrito ${ }^{32}$.

\section{Afectación ganglionar}

La afectación ganglionar produce indudablemente impacto en la supervivencia de los pacientes afectos de CR. La incidencia de ganglios positivos (N1-2) aumenta con el estadio pT, y se asocia con metástasis a distancia y afectación venosa ${ }^{33}$.

Trabajos clásicos, como el de Giberti et al, comunican una alta supervivencia en los $\mathrm{CR} N+$, basándose en la linfadenectomía sistemática. Con ella, consiguen una supervivencia en los N+ MO sin afectación vascular del 53\% a los 5 años, 39\% a los 10 años y $16 \%$ a los 20 años ${ }^{34}$.

Otras publicaciones no conceden una supervivencia mayor al $10 \%$ a los cinco años a estos pacientes $^{35}$.

En 2007 fue publicado un análisis multivariante que incluía 171 pacientes con $\mathrm{CR} N+$ sin metástasis tratados con cirugía, encontrando una supervivencia al año, 2, 5, 10 y 15 años del 71,4\%, 51,6\%, $39,3 \%, 30,5 \%$ y $27,5 \%$ respectivamente, que plantean francamente inferior a los pacientes sin afectación ganglionar ${ }^{36}$.

De esta forma, los pacientes con afectación ganglionar y sin metástasis podrían ser encuadrados en un grupo pronóstico de riesgo intermedio.

\section{Metástasis a distancia}

La diseminación metastásica podríamos calificarla como el peor factor pronóstico. La supervivencia cáncer-específica se situaría entre el 5-10\% a los 5 años y el 0-7\% a los 10 años $^{37}$

\section{FACTORES CLÍNICOS}

En este grupo de factores se incluyen un gran número de variables, no anatomopatológicas, que van desde la esfera de la sintomatología y características del paciente hasta multitud de parámetros analíticos. Intentaremos analizar en este apartado aquellos que poseen, según los estudios más recientes, mayor evidencia pronóstica.

\section{Forma de presentación}

En nuestros días, fundamentalmente debido a la evolución de las técnicas de imagen, el diagnóstico de CR se realiza de forma incidental en torno al $40 \%$ de los $\operatorname{casos}^{17,38}$.

Los tumores diagnosticados de forma incidental presentan una mayor supervivencia: 81\%-97\% vivos a los cinco años frente al 65-75\% de los sintomáticos, diferencia estadísticamente muy significativa $^{17,38-41}$. Tsui et al. ${ }^{42}$, no sólo confirman esta mayor supervivencia sino también un menor porcentaje de recurrencias.

\section{Estado funcional del paciente}

El performance status (PS) es reconocido como factor pronóstico independiente en análisis multivariantes ${ }^{10,42-44}$. Este indicador pronóstico no quedaría limitado a los tumores localmente avanzados y/o metastásicos, sino también para los tumores locali$\operatorname{zados}^{45}$.

El hecho de poseer un PS según la ECOG (Tabla 2) (Eastern Cooperative Oncology Group) mayor a 0 (0: encontrarse totalmente activo, capaz de realizar todas las actividades anteriores a la enfermedad sin restricción), implica un riesgo relativo para el fallecimiento de $3,66^{46}$.

\section{Trombocitosis}

La existencia de trombocitosis es considerada como factor pronóstico independiente, de tal forma que los pacientes con un nivel plaquetario normal presentan mayor supervivencia que aquellos que poseen una cuantificación superior a 400.000/ $\mathrm{mm}^{3,47}$.

Benzalah et al. encuentran una relación directamente proporcional de la tromocitosis con el tamaño tumoral, la invasión ganglionar, la existencia de metástasis y el grado de Fuhrman, e inversamente proporcional a la supervivencia (70\% a los 5 años en aquellos pacientes con nivel plaquetario $<450.000 / \mathrm{mm}^{3}$, frente al $38 \%$ en aquellos con un nivel superior) ${ }^{48}$. 
Tabla 2

\begin{tabular}{ll}
\hline Grado & ECOG \\
\hline 0 & Totalmente activo, capaz de realizar todas las actividades anteriores a la enfermedad sin \\
& restricciones. \\
& Restricciones en las actividades físicamente intensas, pero ambulatorio y capaz de realizar trabajos \\
& de tipo sedentario. \\
& Ambulatorio y capaz de atender todas sus necesidades personales, pero incapaz de realizar \\
& cualquier actividad de trabajo. Levantado hasta y aproximadamente más del 50\% del tiempo de vigilia. \\
& Capaz de atender sus necesidades personales con algunas limitaciones, encamado o sentado más del \\
& $50 \%$ del tiempo de vigilia \\
& Completamente discapacitado. No puede atender ninguna de sus necesidades personales. Totalmente \\
& encamado o sentado. \\
& Muerto.
\end{tabular}

\section{Otros factores clínicos}

Existen múltiples estudios que evalúan otras variables con el objetivo de determinar su influencia en la evolución de la enfermedad, como la pérdida de peso, considerada un factor con implicaciones pronósticas si es superior al $10 \%{ }^{9}$, o la neutrofilia, considerada factor independiente por algunos autores, si situamos el corte en 6.500 neutrófilos por microlitro ${ }^{49}$.

Por último, son considerados, con mayor o menor peso, variables como la anemia, la disfunción hepática, incremento de la velocidad de sedimentación globular, aumento de fosfatasa alcalina, etc.

\section{FACTORES ANATOMOPATOLÓGICOS}

Dentro de estos factores incluimos determinados aspectos histopatológicos que han demostrado tener relevancia en la evolución de la enfermedad, y fundamentalmente con la progresión y supervivencia de la misma. No incluiremos en este apartado la invasión microscópica vascular, por habernos referido a ella anteriormente aunque, obviamente, podría quedar incluido en este grupo de factores pronósticos.

\section{Grado nuclear}

El sistema de gradación nuclear más extendido en la actualidad es el de Fuhrman ${ }^{50}$. Este sistema clasifica a los CR según la morfología nuclear en cuatro grados. En relación a dicho grado nuclear la supervivencia varía de forma significativa, pero con amplias diferencias según las series analizadas; de esta forma la supervivencia a los cinco años puede oscilar de la siguiente forma ${ }^{51}$ :

$\begin{array}{ll}\text { - 50-100\%: Grado } 1 \\ \text { - 30-94\%: } & \text { Grado } 2 \\ \text { - 10-80\%: } & \text { Grado } 3 \\ \text { - 9-66\%: } & \text { Grado } 4\end{array}$
Estas diferencias podrían estar justificadas por varias razones, como son la variabilidad interobservadores, la existencia de más de un grado nuclear en un mismo tumor y las variadas formas y protocolos de procesamiento de las muestras.

No obstante, a pesar de esta disparidad de datos, el grado nuclear ha demostrado ser un factor pronóstico independiente en estudios multivariantes ${ }^{52}$.

\section{Tipo histológico}

El carcinoma cromófobo clásicamente ha sido considerado de mejor pronóstico que el carcinoma de células claras, al igual que los tumores papilares $^{43,53}$. Sin embargo, existen trabajos actuales que encuentran similar supervivencia en los diferentes tipos histológicos, con la salvedad de los que poseen diferenciación sarcomatoide, en los que existe consenso en relación a su peor evolución ${ }^{54,55}$. En este sentido el trabajo publicado el pasado año por Dall`oglio et al. ${ }^{54}$, sitúa la supervivencia libre de enfermedad a los cinco años en el 76,6\% para los tumores de células claras, 71,2\% para los cromófobos y $72,7 \%$ para los papilares, descendiendo hasta el $26,9 \%$ para los que poseen diferenciación sarcomatoide.

\section{Necrosis tumoral}

La presencia de necrosis en el CR ha sido considerada un importante factor predictor de supervivencia, asociándose a mayor tamaño tumoral, afectación ganglionar y metástasis, así como grados indiferenciados y peor performance status. La supervivencia cáncer-específica a los cinco años desciende del $75 \%$ en aquellos tumores sin necrosis hasta el $36 \%$ en los que la poseen ${ }^{56}$. 


\section{FACTORES MOLECULARES}

La incorporación de nuevas tecnologías en estudios genéticos, proteinómica y de $\mathrm{ADN}$ han abierto un amplio horizonte en el campo de la tumorogénesis y progresión del CR. Por otro lado, el desarrollo de nuevos tratamientos, basados en dianas terapéuticas, ha estimulado la búsqueda de marcadores moleculares; estos marcadores podrían facilitar la estratificación del riesgo de los tumores renales.

Actualmente han demostrado, en análisis multivariante, ser factores pronósticos independientes para el $\mathrm{CR}^{57}$ :

- Inductores de hipoxia: CA IX (carbonic anhydrase IX) y VEGF (vascular endotelial growth factor).

- Factor de proliferación Ki67.

- Regulador del ciclo celular p53.

La CA IX juega un importante papel en la regulación del pH celular, y se ha comprobado que más del $80 \%$ de los CR primarios y metastásicos la expresan, a diferencia del riñón normal ${ }^{58}$. Por tanto, se considera un factor de mal pronóstico.

Ki67 es un antígeno de expresión nuclear expresado en las fases G1, G2, G3 y M del ciclo celular, pero no en la G0, por lo tanto, evalúa la proliferación celular (63-Onda). Por ello, un índice proliferativo elevado determinado mediante la expresión de Ki67 se asociaría a estadíos avanzados, pobre diferenciación histológica y mal pronóstico. De esta forma una expresión elevada se asociaría a una supervivencia cáncer-específica a los cinco años de 6\%, frente al $79 \%$ en aquellos tumores que lo expresan poco ${ }^{61}$.

El oncogén p53, regulador del ciclo celular mediante la producción de la proteína p53, es capaz de producir una parada de dicho ciclo en su fase G1, permitiendo la reparación del ADN o estimulando la apoptosis de células dañadas. Este factor ha sido discutido como factor pronóstico en el CR, pero parece que recientes estudios demuestran sus implicaciones pronósticas $^{32,57}$. Las mutaciones de dicho gen favorecerían la progresión de tumor.
Otros factores moleculares, estudiados en múltiples trabajos, que han presentado implicaciones pronósticas, en mayor o menor grado, son el VHL, HIF, PTEN, CD44, E Cadherina. Por otro lado, se perfilan como futuros marcadores moleculares el CD 10, parvalbumina, AMACR, CK7 y S100A1, cuyo papel pronóstico aún no ha sido bien evaluado ${ }^{32}$.

\section{NOMOGRAMAS PREDICTIVOS}

En la actualidad disponemos de modelos pronósticos elaborados por diferentes grupos de trabajo para pacientes afectos de CR, cuyo objetivo es facilitar su manejo y orientación terapéutica adyuvante. Estos nomogramas o algoritmos integran un número determinado de factores pronósticos (anatómicos, clínicos e histológicos), obteniendo resultados predictivos en relación a la evolución de la enfermedad. Intentaremos exponer de forma resumida algunos de estos nomogramas.

El grupo del Memorial Sloan-Kettering Cancer Center (MSKCC) propone un nomograma (Fig. 1) para los pacientes con CR de células claras localizado, que incluye el tamaño tumoral, estadio tumoral $\mathrm{T}$, grado, existencia de necrosis, invasión vascular y forma de presentación, con el que predice la probabilidad de encontrarse libre de enfermedad a los 5 años $^{44}$.

En la Universidad de California, los Ángeles (UCLA) han elaborado un modelo (Integarate Staging System-UISS) que clasifica a los pacientes en grupos de riesgo con diferencias estadísticamente significativas en cuanto a la supervivencia cáncer-específica.

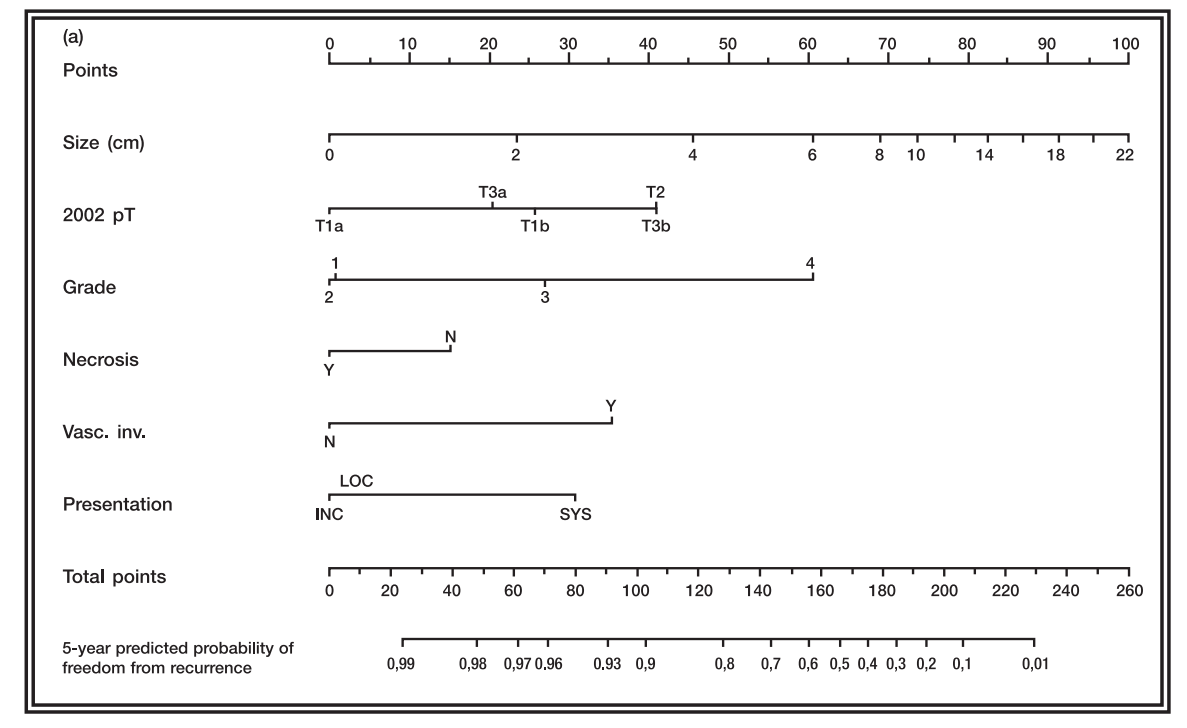

FIGURA 1. Nomograma Memorial Sloan-Kettering Cancer Center (MSKCC). 
Este modelo integra el estadio T, el grado y el performance status (ECOG), diferenciando metastáticos de no metastáticos, con lo que consigue agrupar a los pacientes en riesgo bajo, intermedio o alto (Fig. 2). Para los no metastásicos ofrece una supervivencia a los 5 años del 83,8\%, 71,9\% ó 44\% según sean de bajo, intermedio o alto riesgo respectivamente; mientras que para los metastáticos sería del 30\%, 19,3\% o 0\% según se clasifiquen como bajo, intermedio o alto riesgo respectivamente ${ }^{45,46}$.

En The Mayo Clinic han desarrollado un nomograma que predice la supervivencia, tras calcular un score del 1 al 10 (SSING), desde el primero hasta el décimo año (Fig. 3). Este modelo integra el estadio, tamaño, grado y la existencia de necrosis ${ }^{64}$.

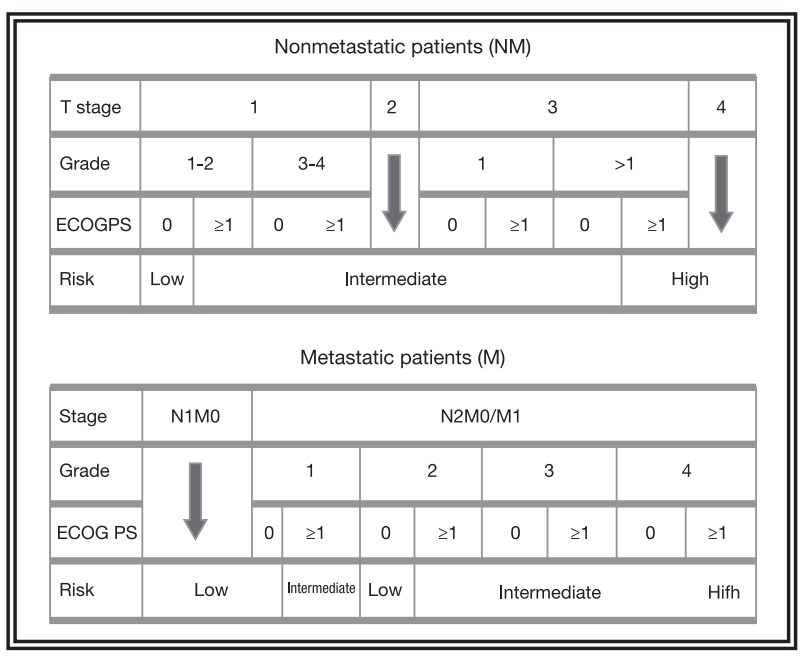

FIGURA 2. UISS (Integarate Staging System), Universidad de California (UCLA).
En relación a los $\mathrm{CR}$ metástasicos, Motzer et al establecen un nomograma, basado en el performance status según la escala de Karnofsky, siendo diferenciador si éste se sitúa por debajo del $80 \% 65$.

En 2003, Cindolo et al. publicaron una fórmula matemática (RRF: recurrence risk formula) (Fig. 4) para el cálculo de recurrencia en pacientes no metastáticos, basada, tras un análisis uni y multivariante que incluía diferentes factores pronósticos, en la forma de presentación (sintomática o no) y el tamaño tumoral en $\mathrm{cm}$. Si el RRF es =1,2 la probabilidad de supervivencia libre de enfermedad a los dos y cinco años sería del 96 y 93\% respectivamente, mientras que si este índice es superior a 1,2, esta probabilidad descendería al 83 y $68 \%$ respectivamente ${ }^{66}$.

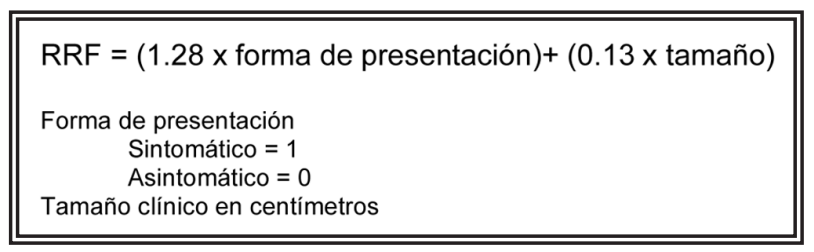

FIGURA 4. Fórmula RRF (recurrence risk formula).

Estos modelos matemáticos han sido evaluados ${ }^{67}$, otorgándoles mayor o menor grado de fiabilidad. Lo cierto es que la Guía Europea del Carcinoma de células renales no recomienda en la actualidad el uso rutinario de nomogramas o modelos predictivos ${ }^{68}$, y además, aún queda por delante el integrar en estos modelos los factores moleculares que, como hemos visto, parecen tener un importante papel en la determinación del pronóstico del CR.

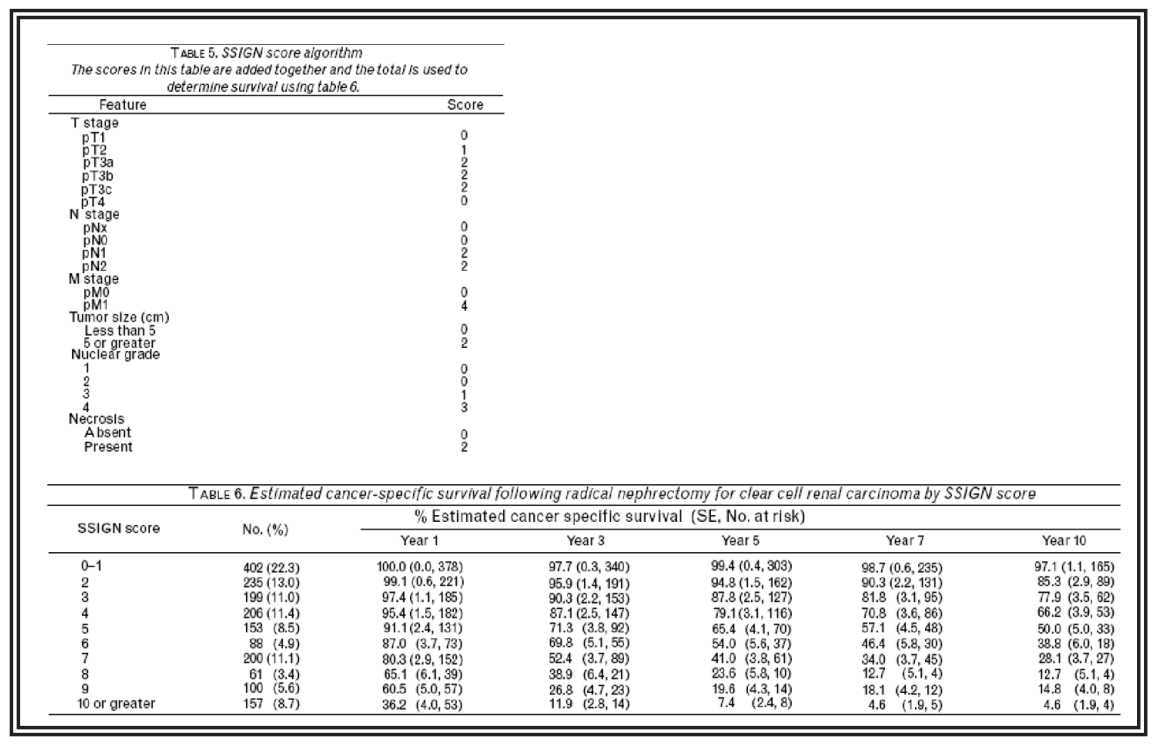

FIGURA 3. Score SSING - Mayo Clinic.

\section{REFERENCIAS}

1. La situación del cáncer en España. Ministerio de Sanidad y Consumo. Madrid, 2005. ISBN: 84-7670-673-681.

2. Berrino F, De Angelis R, Sant M, Rosso S, Bielska-Lasota M, Coebergh JW et al. Survival for eight major cancers and all cancers combined for European adults diagnosed in 1995-1999: results of the EUROCARE-4 study. Lancet Oncol 2007;8(9):773-783.

3. Kirkali Z, Tuzel E, Mungan MU. Recent advances in kidney cancer and metastatic disease. BJU Int. 2001;88(8):818824.

4. López-Beltran A, Scarpelli M, Montironi R, Kirkali Z. 2004 WHO classification of the renal tumors of the adults. 2006;49(5):798-805. Epub 2006 Jan 17.

5. Ljungberg B. Prognostic markers in renal cell carcinoma. Curr Opin Urol. 2007; 17(5):303-308 
6. Lane BR, Kattan MW. Predicting outcomes in renal cell carcinoma. Curr Opin Urol. 2005;15(5):289-297.

7. Belldegrun AS. Renal Cell Carcinoma: Prognostic Factors and Patient Selection. Eur Urol Supp. 2007;6:477-483.

8. AJCC. Cancer staging Manual, Sixth edition (2002). www. springer-ny.com/medicine.

9. Tsui KH, Shvarts O, Smith RB, et al. Prognostic indicators for renal cell carcinoma: a multivariate analysis of 643 patients using revised 1997 TNM staging criteria. J Urol 2000; 163(4): 1090-1095.

10. Park WH, Eisen T. Prognostic factors in renal cell cancer. BJU Int 2007 May;99(5 Pt B):1277-1281.

11. Patard JJ, Shvarts O, Lam JS, Pantuck AJ, Kim HL, Ficarra V et al. Safety and efficacy of partial nephrectomy for all T1 tumors based on an international multicenter experience. $\mathrm{J}$ Urol 2004;171(6 Pt 1):2181-2185.

12. Knight DA, Stadler WM. Prognostic factors in localized renal cell cancer. BJU Int 2007 May;99(5 Pt B):1212-1218.

13. Frank I, Blute ML, Leibovich BC, Cheville JC, Lohse CM, Zincke H. Independent validation of the 2002 American Joint Committee on cancer primary tumor classification for renal cell carcinoma using a large, single institution cohort. J Urol. 2005; 173(6): 1889-1992.

14. Leibovich BC, Blute ML, Cheville JC, Lohse CM, Weaver AL, Zincke H Nephron sparing surgery for appropriately selected renal cell carcinoma between 4 and $7 \mathrm{~cm}$ results in outcome similar to radical nephrectomy. J Urol 2004;171(3):1066-1070.

15. Ficarra V, Guillè F, Schips L, de la Taille A, Prayer Galetti T, Tostain J et al. Proposal for revisión of the TNM classificarion system for renal cell carcinoma. Cancer 2005;104(10):2116-2123.

16. Ficarra V, Prayer-Galetti T, Novara G, Bratti E, Zanolla L, Da Bianco $\mathrm{M}$ et al. Tumor-size breakpoint for prognostic stratification of localized renal cell carcinoma. Urol 2004;63(2):23652369; discussion 239-240.

17. Ortiz Gorraiz M, Vicente Prados FJ, Rosales Leal JL, Honrubia Vílchez B, Martínez Morcillo A, Cózar Olmo JM et al. Valoración de factores pronósticos de la supervivencia en una serie de 202 pacientes inervenidos por carcinoma de células renales. Actas Urol Esp 2005;29(2):179-189.

18. Budía Alba A, Gómez Pérez L, Bango V, Ruiz-Cerdá JI, Sempere A, Queipo JA, et al. Análisis de los factores pronósticos de progresión tumoral en el adenocarcinoma renal. Actas Urol Esp. 2007;31(8):831-844.

19. Medina López RA, Congregado Ruiz CB, Campoy Martínez P, Morales López A, Sánchez Gómez E, pascual del Pobil Moreno JL. Cáncer renal: análisis descriptivo de una serie de 267 casos intervenidos. Arch Esp Urol. 2001;54(5):423-428.

20. Giménez Bachs JM, Donate Moreno MJ, Salinas Sánchez AS, Pastor Navarro H, Carrión López P, Pastor Guzmán JM, et al. Supervivencia en relación a los factores pronósticos en una serie de pacientes con carcinoma de células renales. Arch Esp Urol. 2007;60(10):1167-1174.

21. Dall'Oglio MF, Ribeiro-Filho LA, Antunes AA, Crippa A, Nesrallah L, Gonçalves PD, et al. Microvascular tumor invasion, tumor size and Fuhrman grade: a pathological triad for profnostic evaluation of renal cell carcinoma. J Urol 2007;178 (2):525-428.

22. Klatte T, Chung J, Leppert JT, Lam JS, Pantuck AJ, Figlin RA, et al. Prognostic relevance of capsular involvement and collecting sytem invasion in stage I and II renal cell carcinoma. BJU Int. 2007;99(4): 821-824.

23. Han KR, Bui MH, Pantuck AJ, Freitas DG, Leibovich BC, Dorey FJ et al. TNM T3a renal cell carcinoma: adrenal gland involvement is not the same as renal fat invasion. J Urol. 2003;169(3): 899-903.
24. Van Poppel H, Vandendriessche H, Boel K, Mertens V, Goethuys H, Haustermans K, et al. Microscopic vascular invasions ins the most relevat prognosticator after radical nephrectomy for clinicalli nonmetastatic renal cell carcinoma. J Urol. 1997; 158(1):45-49.

25. Lang H, Lindner V, Letourneux H, Martin M, Saussine C, Jacqmin D, et al. Prognostic value of microscopic venous invasion in renal cell carcinoma: long-term follow-up. Eur Urol. 2004;46(3):331-335.

26. Ishimura T, Sakai I, Hara I, Eto H, Miyake H. Microscopic venous invasion in renal cell carcinoma as a predictor of recurrence after radical surgery. Int J Urol. 2004;11(5):264-268.

27. Madbouly K, Al-Qahtani SM, Ghazwani Y, Al-Shaibani S, Mansi MK. Microvascular tumor invasion: prognostic significance in low-stage renal cell carcinoma. Urology. 2007;69(4): 670-674.

28. Kim HL, Zisman A, Han KR, Figlin RA, Belldegrun AS. Prognostic significance of venous thrombus in renal cell carcinoma. Are renal vein and inferior vena cava involvement different?. J Urol. 2004;171(2 Pt 1):588-591.

29. Moinzadeh A, Libertino JA. Prognostic significance of tumor thrombus level in patients with renal cell carcinoma and venous tumor thrombus extension. Is all T3b the same?. J Urol. 2004; 171(2 Pt 1):598-601.

30. Lambert EH, Pierorazio PM, Shabsigh A, Olsson CA, Benson MC, McKiernan JM. Prognostic risk stratification ans clinical outcomes in patients indergoing surgical treatment for renal cellc carcinoma with vascular tumor thrombus. Urology. 2007;69(6): 1054-1058.

31. Boorjian SA, Sengupta S, Blute ML. Renal cell carcinoma: vena caval involvement. BJU Int. 2007; 99(5 Pt B):1239-1244.

32. Ficarra V, Galfano A, Verhoest G. Prognostic Factors and Staging Systems for Renal Cell Carcinoma. Eur Urology supp. 2007; 6: 623-629.

33. Pantuck AJ, Zisman A, Dorey F, Chao DH, Han KR, Said J, et al. Renal cell carcinoma with retroperitoneal limph nodes. Impact on survival an benefits of immunotherapy. Cancer. 2003;97(12):2995-3002.

34. Giberti C, Oneto F, Martorana G, Rovida S, Carmignani G. Radical nephrectomy for renal cell carcinoma: long-term results and prognostic factors on a series of 328 cases. Eur Urol. 1997;31(1):40-8.

35. Lam JS, Shvarts O, Leppert JT, Figlin RA, Belldegrun AS. Renal cell carcinoma 2005: new frontiers in staging, prognostication and targeted molecular therapy. J Urol. 2005;173(6):18531862 .

36. Karakiewicz PI, Trinh QD, Bhojani N, Bensalah K, Salomon L, de la Taille A, et al. Renal Cell Carcinoma with Nodal Metastases in the Absence of Distant Metastatic Disease: Prognostic Indicators of Disease-Specific Survival. Eur Urol. 2007;51(6): 1616-1624.

37. Lohse CM, Cheville JC. A review of prognostic pathologic features and algorithms for patients treated surgically for renal cell carcinoma. Clin Lab Med. 2005;25(2):433-464.

38. Congregado Ruiz B, Medina López RA, Sánchez Gómez E, Morales López A, Pascual del Pobil JL. Diagnóstico incidental del carcinoma renal. ¿implica un mejor pronóstico?. Actas Urol Esp. 2001;25(4):278-282.

39. Siemer S, Uder M, Humke U, Lindenmeier T, Moll V, Rüdenauer E, et al. Value of ultrasound in early diagnosis of renal cell carcinoma. Urologe A. 2000;39(2):149-153.

40. Lightfoot N, Conlon M, Kreiger N, Bissett R, Desai M, Warde P, et al. Impact of non-invasive imaging on increased incidental detection of renal cell carcinoma. Eur Urol. 2000;37(5):521527. 
41. Pantuck AJ, Zisman A, Belldegrum AS. The changinh natural history of renal cell carcinoma. J Urol. 2001;166(5):1611-1623.

42. Tsui KH, Shvarts O, Smith RB, Figlin R, de Kernion JB, Belldegrun A. Renal cell carcinoma: prognostic significance of incidentally detected tumors. J Urol. 2000;163(2):426-430.

43. Frank I, Blute ML, Cheville JC, Lohse CM, Weaver AL, Zincke H. An outcome prediction model for patients with clear cell renal cell carcinoma treated with radical nephrectomy based on tumos stage, size, grade and necrosis: the SSIGN score. J Urol. 2002;168(6):2395-2400.

44. Kattan MW, Reuter V, Motzer RJ, Katz J, Russo P. A prospective prognistic nomogram for renal cell carcnoma. J Urol. 2001;166(1):63-67.

45. Zisman A, Pantuck AJ, Dorey F, Said JW, Shvarts O, Quintana D, et al. Improved prognostic of renal cell carcinoma using and integrated staging system. J Clin Oncol. 2001;19(6):16491657.

46. Zisman A, Pantuck AJ, Dorey F, Chao DH, Gitlitz BJ, Moldawer $\mathrm{N}$, et al. Mathematical Model to Predict Individual Survival for Patients With Renal Cell Carcinoma. J Clin Oncol. 2002;20(5): 1368-1374.

47. O’Keefe SC, Marshall FF, Issa MM, Harmon MP, Petros JA. Thrombocytosis is associated with a significant increase in the cancer specific death rate after radical nephrectomy. J Urol. 2002;168(4 Pt 1):1378-1380.

48. Bensalah K, Leray E, Fergelot P, Rioux-Leclercq N, Tostain J, Guillé $\mathrm{F}$, et al. Prognostic value of thrombocytosis in renal cell carcinoma. J Urol. 2006;175(3 Pt 1):859-863.

49. Atzpodien J, Royston P, Wandert T, Reitz M; DGCIN - German Cooperative Renal Carcinoma Chemo-Immunotherapy Trials Group. Metastatic renal carcinoma comprehensive prognostic system. Br J Cancer. 2003;88(3):348-353.

50. Fuhrman SA, Lasky LC, Limas C. Prognostic significance of morphologic parameters in renal cell carcinoma. Am J Surg Pathol. 1982;6(7):655-663.

51. Novara G, Martignoni G, Artibani W, Ficarra V. Grading systems in renal cell carcinoma. J Urol. 2007;177(2):430-438.

52. Rioux-Leclercq N, Leray E, Fergelot P, et al. The Fuhrman grade: a European multicenter study for the determination of the optimal grading system in term of prognosis. Eur Urol suppl. 2006; 5: 67-70.

53. Mejean A, Oudard S, Thiounn N. Prognostic factors of renal cell carcinoma. J Urol. 2003;169(3):821-827.

54. Dall`Oglio M, Aap MA, Antunes AA, Cury J, Leite KR, Srougi M. Impact of clinicopathological parameters in patients treated for renal cell carcinoma. J Urol. 2007;177(5):1687-1691.

55. Patard JJ, Leray E, Rioux-Leclercq N, Cindolo L, Ficarra V, Zisman A, et al. Prognostic value of histologic subtypes in renal cell carcinoma: a multicenter experience. J Clin Oncol. 2005; 23(12):2763-2771.

56. Lam JS, Shvarts O, Said JW, Pantuck AJ, Seligson DB, Aldridge $\mathrm{ME}$, et al. Clinicopathologic and molecular correlations of necrosis in the primary tumor of the primary tumor patients with renal cell carcinoma. Cancer. 2005;103(12):25172525 .
57. Martignoni G, Brunelli M, Gobbo S, Remo A, Ficarra V, CossuRocca $\mathrm{P}$, et al. Role of molecular markers in diagnosis and prognosis of renal cell carcinoma. Anal Quant Cytol Histol. 2007;29(1):41-49.

58. Bui MH, Seligson D, Han KR, Pantuck AJ, Dorey FJ, Huang Y, et al. Carbonic anhydrase IX is an independent predictor of survival in advanced renal clear cell carcinoma: implications for prognosis and therapy. Clin Cancer Res. 2003;9(2):802811.

59. Jacobsen J, Grankvist K, Rasmuson T, Ljungberg B. Different isoform patterns for vascular endothelial growth factor between clear cell and papillary renal carcinoma. BJU Int. 2006;97(5): 1102-1108.

60. Ljungberg BJ, Jacobsen J, Rudolfsson SH, Lindh G, Grankvist $\mathrm{K}$, Rasmuson T. Diferent vascular endothelial growth factor (VEGF), VGF-receptor 1 and 2 mRNA expression profiles between clear cell and papillary renal cell carcinoma. BJU Int. 2006;98(3):661-667.

61. Yildiz E, Gokce G, Kilicarslan H, Ayan S, Goze OF, Gultekin EY. Prognostic value of the expression of Ki-67, CD44 and vascular endothelial growth factor, and microvessel invasion, in renal cell carcinoma. BJU Int. 2004;93(7):1087-1093.

62. Lam JS, Leppert JT, Yu H, et al. Expression of the vascular endothelial growth factor family in tumor dissemination and disease free survival in clear cell renal cell carcinoma. J Clin Oncol. 2005;23(Suppl):387s (abstract no. 4538).

63. Onda H, Yasuda M, Serizawa A, Osamura RY, Kawamura N, et al. Clinical outcome in localized renal cell carcinoma related to immunoexpression of proliferating cell nuclear antigen, Ki-67 antigen, and tumor size. Oncol Rep. 1999;6(5):1039-1043.

64. Frank I, Blute ML, Cheville JC, Lohse CM, Weaver AL, Zincke H. An outcome prediction model for patients with clear cell renal cell carcinoma treated with radical nephrectomy based on tumor stage, size, grade and necrosis: the SSIGN score. J Urol. 2002;168(6):2395-2400.

65. Motzer RJ, Bacik J, Schwartz LH, Reuter V, Russo P, Marion S, et al. Prognostic factors for survival in previously treatted patient with metastatic renal cell carcinoma. J Clin Oncol. 2004;22(3):454-463.

66. Cindolo L, de la Taille A, Messina G, Romis L, Abbou CC, Altier $\mathrm{V}$, et al. A prospective clinical prognostic model for non-metatatic renal cell carcinoma. BJU Inte. 2003;92(9):901-905.

67. Karakiewicz PI, Hutterer GC. Predicting cancer-control outomes in patients with renal cell carcinoma. Curr Opin Urol. 2007;17(5):295-302.

68. Ljungberg B, Hanbury DC, Kuczyk MA et al. Guidelines on Renal Cell Carcinoma 2007. www.uroweb.org.

Correspondencia autor: Dr. Rafael A. Medina López

Servicio de Urología. Hospital Universitario Virgen del Rocío

Av. Manuel Siurot s/n - 41013 Sevilla. Tel.: 955012352

E-mail autor: rantonio.medina.sspa@juntadeandalucia.es

Información artículo: Original

Trabajo recibido: marzo 2009

Trabajo aceptado: abril 2009 